\title{
SEMBLANZA DEL DR. D. ENRIQUE PÉREZ CASTRO EN EL CENTENARIO DE SU NACIMIENTO (1908-1980)
}

\author{
Mariano Pérez Albacete.
}

Servicio de Urología. Hospital Universitario Virgen de la Arrixaca. Murcia. España.

\begin{abstract}
Resumen.- OBJETIVO: Con ocasión de cumplirse el centenario del nacimiento del Dr. D. Enrique Pérez Castro, figura relevante en nuestra especialidad en la segunda mitad del siglo XX, realizamos un estudio del contexto urológico en el que se desarrolló su existencia y recogemos los principales datos sobre su vida y obra con la finalidad de rendir un reconocimiento a su memoria como gran personalidad de la Urología.
\end{abstract}

MÉTODOS: Revisamos los libros de Historia de la Urología, sus escritos y la publicación Archivos Españoles de Urología durante el periodo de su dirección y cuantas referencias hemos encontrado en relación con él.

RESULTADO/CONCLUSIÓN: Persona de brillante currículo académico, se preparó en la especialidad de Urología en los mejores centros. Residió en Madrid, su ciudad natal, en la que transcurrió toda su carrera profesional, en el Hospital Provincial, del que fue jefe del servicio de Urología, y en la Universidad Central donde fue profesor agregado de la asignatura. Estudioso, con espíritu investigador, sus trabajos, de gran calidad y por los que recibió varios premios, están expuestos con claridad y con un depurado estilo y siempre acompañados de una extensa bibliografía. Resaltamos su mérito principal, la fundación de la publicación Archivos Españoles de Urología, fruto de su temperamento, de su capacidad organizadora e inquietud científica, revista que editó y dirigió a lo largo de 36 años con ilusión y dedicación y, gracias a su tesón, logró que se consolidara como el referente de la Urología española.

Palabras clave: Historia de la Urología. Biografías urológicas. Enrique Pérez Castro.

Summary.- OBJECTIVES: With the occasion of the Centenary of Dr. Enrique Perez Castro's birth, being a relevant figure in our speciality in the second half of the 20th century, we performed a study of the urological environment in which his life was developed and collect the main data about his life and works with the aim of paying tribute to his memory as a great personality in urology.

METHODS: We review the books of History of Urology, his writings and the journal Archivos Españoles de Urologia during the period of his directorship and as many references related to him as we found.

RESULTS/CONCLUSIONS: He had a brilliant academic curriculum; he trained urology in the best centres. He lived in Madrid, his birth place, were his entire professional career developed, in the Hospital Provincial, where he was the chairman of urology, and the Central University where he was associated professor. He liked 
to study, and had researcher spirit; his works, which had great quality -he received several prizes-, are clearly exposed, with a fine style, always with an extensive bibliography. We emphasize his main merit, the foundation of the journal Archivos Españoles de Urología, a fruit of his personality, his organizing capacity and scientific restlessness, of which he was editor and directed with eagerness and dedication during 36 years and, thanks to his tenacity, he consolidated it as the reference of the Spanish urology.

Keywords: History of urology. Urological biographies. Enrique Perez Castro.

\section{INTRODUCCIÓN}

Nació en Madrid el 4 de noviembre de 1908 (Figura 1), hiio del también urólogo Valentín Enrique Pérez Grande ${ }^{1}$ (Figura 2), tras realizar el bachillerato en el instituto Cardenal Cisneros de Madrid, se decantó por los estudios de Medicina que llevó a cabo en la Universidad Central, donde fue alumno interno de Patología Quirúrgica con el Dr. Rafael Mollá Rodrigo ${ }^{2}$. En 1931 obtuvo el grado de licenciado con la nota de sobresaliente. Ingresó mediante concurso como médico interno en la Escuela de Urología de la Casa de Salud de Valdecilla ${ }^{3}$, permaneció allí por espacio de dos años y logró el premio de su creador, el marqués de Pelayo, por la labor realizada; prepara allí su memoria de tesis doctoral, La anestesia con evipán sódico en Urología (Figura 6), que fue dirigida por su mentor el Dr. Picatoste, leída en Madrid en 1934 y calificada con sobresaliente.

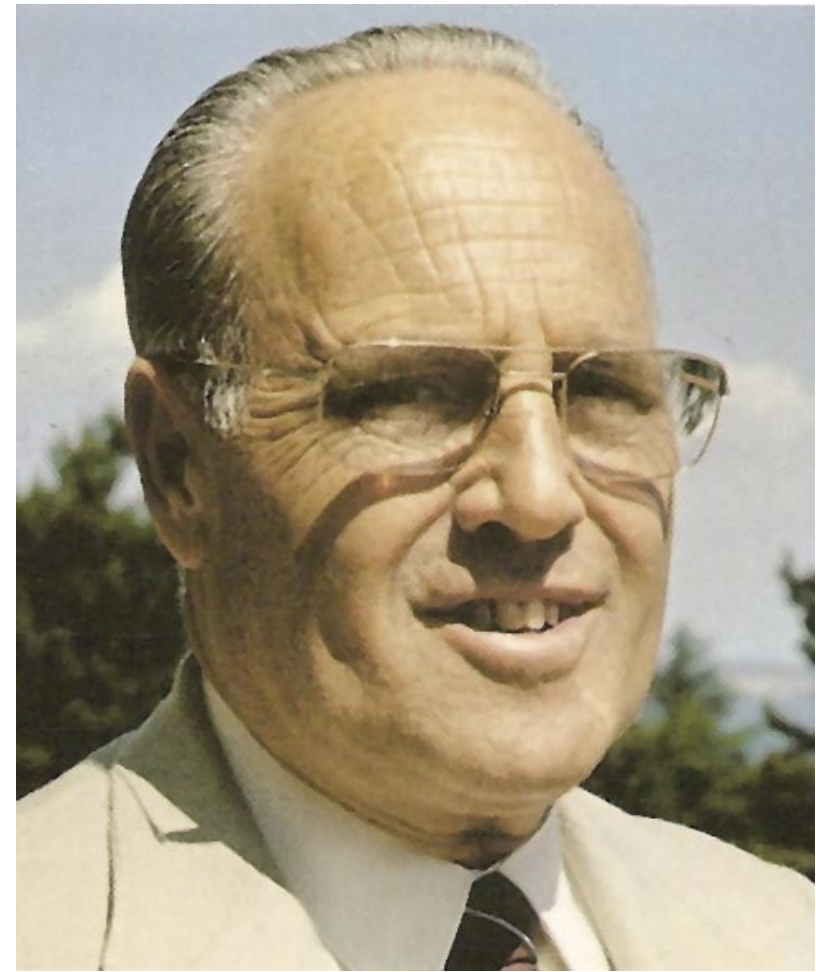

FIGURA 1. Dr. D. Enrique Pérez Castro (1908-1980).

Una vez finalizada su estancia en la capital cántabra regresó a Madrid donde, por un corto periodo, estuvo como médico agregado en el Instituto del Cáncer. Logró una beca de la Junta de Ampliación de Estudios e Investigaciones Científicas con la que prosiguió su especialización en Berlín como asistente de Urología en la clínica urológica regentada por el profesor Alexander von Lichtenberg ${ }^{4}$, donde se iniciaron las exploraciones radiológicas tras la

1.- D. Valentín Enrique Pérez Grande (1878-1968) fue colaborador del profesor libre de Urología en la Universidad Central, Dr. Alberto Suárez de Mendoza (n. 1849), al que ayudó con la trascripción de sus clases, lo que dio origen al segundo tratado urológico redactado en español, Diagnóstico y tratamiento de las enfermedades de las Vías Urinarias, (Madrid, 1908) (Figura 3); fue también uno de los fundadores de la Asociación Española de Urología, de la que fue secretario de actas en su segunda junta directiva, en 1914, y vocal hasta 1923. Creó la publicación Conferencias Clínicas de Urología (Figura 4), dirigida al médico general y editada en Madrid, de 1919 a 1936, con 162 números en los que recopiló todos sus trabajos y en los que repasó las enfermedades y procedimientos diagnósticos y terapéuticos urológicos y venéreos con la finalidad de difundir el conocimiento de estas patologías.

2.- D. Rafael Mollá Rodrigo (1862-1930), pionero de la especialidad y autor del primer tratado de Urología escrito en nuestro país, Resumen práctico de diagnóstico y técnica médico quirúrgica de la afecciones de las Vías urinarias (Valencia, 1894) (Figura 5).

3.- D. Ramón Pelayo de la Torriente, Marqués de Pelayo, fundó en Santander La Casa de Salud de Valdecilla, complejo hospitalario modélico y de gran prestigio nacional, donde creó un Instituto de Postgrado en el que el Dr. Julio Picatoste Picatoste (1890-1965) como jefe del servicio de Urología organizó, en 1929, una Escuela de Urología, que alcanzó fama y donde se formó un nutrido grupo de urólogos españoles.

4.- Alexander von Lichtenberg (1880-1949), de origen judío y húngaro de nacimiento, una de las personalidades alemanas de mayor autoridad, jefe del servicio de Urología del Sankt Hedwigs Krankenhaus, de Berlín, donde, en 1906, realizó las primeras pielografías ascendentes tras introducir un contraste, el "Colargol", a través de un catéter ureteral junto con el Dr. Friedrich Voelcker, (1872-1955), profesor de la Universidad de Heidelberg, quien además contribuyó a la Urología con la introducción de la cromocistoscopia en 1903 y la cistografía retrógrada en 1905. En 1929, Lichtenberg encargó al Dr. Moses Swick (n. 1900) norteamericano que disfrutaba de una beca en su servicio, ensayar la inyección intravenosa de un contraste yodado, el "Uroselectan" con el resultado de su eliminación renal y visualización de la vía excretora urinaria, de este modo nació la urografía, publicó el ensayo en inglés por lo que los americanos le consideran su descubridor. Lichtenberg sufrió persecución por los nazis por lo que emigró a Méjico, donde falleció. 


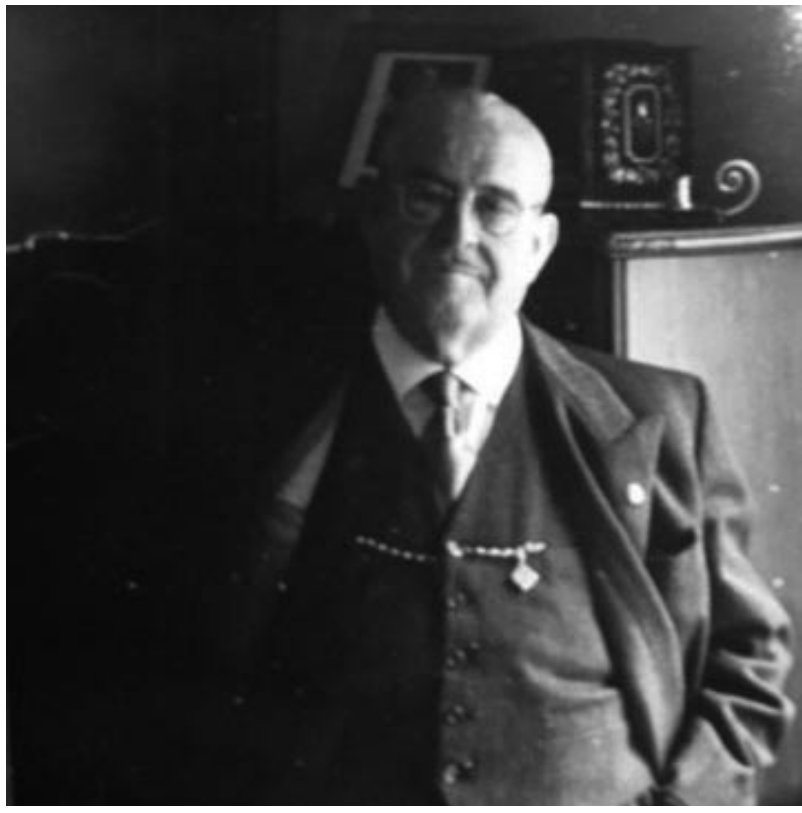

FIGURA 2. Dr. D. Valentín Enrique Pérez Grande (1878-1968). Padre del Dr. Pérez Castro.

inyección de contrastes yodados que permitieron conocer no sólo la morfología renal y la estructura de sus cavidades sino también la recuperación funcional del riñón, además de estudiar su patología, todo ello imposible de conseguir hasta ese momento, lo que posibilitaba también realizar la cirugía conservadora defendida por el profesor Lichtenberg; en este ambiente de trabajo consolidó su preparación que continuó más adelante pensionado por la Fundación "San Nicolás" de la Real Academia de Medicina en Friburgo como asistente en el Instituto de Patología del profesor Büchner.

Al iniciarse la Guerra Civil regresó a España y se incorporó como cirujano al ejército nacional; destinado al Hospital Militar de Zaragoza como teniente médico primer ayudante del Dr. Manuel González

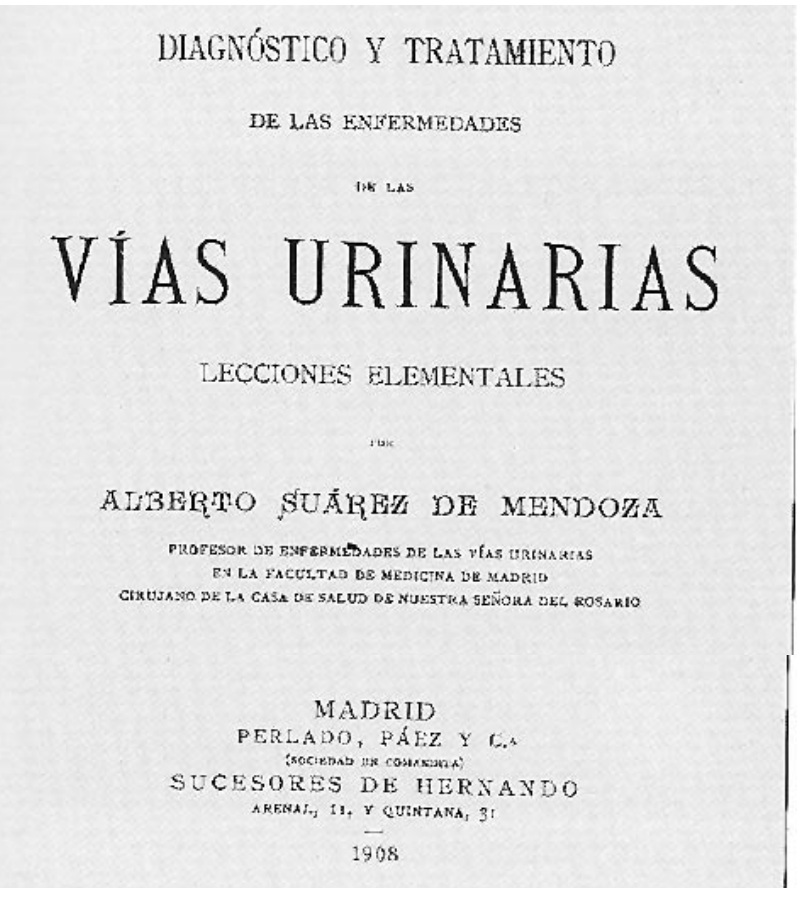

FIGURA 3. Diagnóstico y tratamiento de las enfermedades de las Vías Urinarias, (Madrid, 1908) segundo tratado de Urología escrito en España por el Dr. Suárez de Mendoza.

Ralero, profesor suyo en la Facultad de Medicina, con quien adquirió habilidad y destreza quirúrgicas, estuvo en el frente, en la batalla de Teruel, donde operaba sin descanso hasta que, al rendirse la ciudad, en enero de 1938, fue hecho prisionero y continuó en cautiverio hasta el final de la contienda. Le fue concedida la Medalla de la Campaña, la de Sufrimientos por la Patria y la Cruz del Mérito Militar de primera clase.

Con la llegada de la paz, ingresa en 1939 de ayudante en el servicio de Urología del Hospital de la Beneficencia Provincial de Madrid ${ }^{5}$, al que ha-

5.- Centro regido y administrado por la Diputación desde 1849 fue el segundo nosocomio del país en contar con un servicio de Urología que fue creado en 1889 gracias al diputado provincial Dr. Pulido Fernández, quien, con su Reglamento del Cuerpo Médico-Farmacéutico de los Hospitales Provinciales de Madrid, logró la introducción en los servicios de cirugía de diferentes ramas de especialidades quirúrgicas, entre las que se encontraba la de Vías Urinarias y facilitó también el que se otorgara capacidad docente a estos centros así como la concesión de título de especialista a los médicos allí formados; fue encargado para desempeñar la asistencia genitourinaria el Dr. Alfredo Rodríguez Viforcos (1854-1903), cirujano del hospital de gran prestigio y dedicación especial a esta patología. Le sucedió, en 1904, el Dr. Antonio Bravo Piqueras (1856-1914), excelente clínico y hábil operador entregado por entero a su profesión y cuya hija, religiosa de San Vicente, Sor Ángela, fue años más tarde supervisora en el servicio de Urología.

En 1914 se hizo cargo de la jefatura, por oposición, el Dr. Isidro Sánchez Covisa (1879-1944), que había cursado estudios de la especialidad en el Hospital Necker de París y en Londres; aunque el hospital carecía de recursos, con su habilidad, su poder de persuasión y su gran capacidad organizadora comenzó una paulatina transformación del servicio de Urología hasta lograr la ampliación y modernización de sus quirófanos y equiparlo con los mejores medios de exploración (Figura 8), de tal modo que llegó a convertirlo, en 1931, en el de mayor calidad y prestigio de España, equiparable a los mejores del extranjero y al que acudían a formarse en la especialidad multitud de médicos, muchos de ellos hispanoamericanos; toda la labor realizada anvalmente por sus miembros queda recogida en la publicación Urología Clínica, boletín anual editado por él entre 1928 y 1936; al término de la Guerra Civil por motivos políticos se exiló a Sudamérica y pudo regresar en 1942. 


\section{CONFERENCIAS CLINICAS OE UROLCOIA}

REVISTA MENSUAL

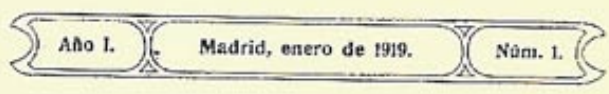

\section{A NUESTROS LECTORES}

Consecuentes con la conducta que cenimos sigutendo hace yn mas de quince años, de hacer vulgariatacion de la especialidad de enfermedades de las vias urinarias, especialidad tan desconocidla, a la vez que tan necesaria para el médico general, oamos holy a inaugurar una serie de Conferencias turodologicictus, que, acaso inmodestamente, consideramos de une intportancia extraordinaria para el médico eminente. mente práctico.

Damatua. In. .......

FIGURA 4. Conferencias clínicas de Urología, editada por el Dr. Valentín Enrique Pérez Grande de 1919 a 1936.

bía sido llamado para dirigir el servicio el Dr. Ángel Pulido Martín (1878-1970), uno de los patriarcas de la Urología española de extraordinario currículo urológico, que introdujo la exploración cistoscópica y el caterismo ureteral en nuestro país; encontró destrozada la unidad y tuvo que comenzar a rehacerla.

En 1940 el Dr. Pérez Castro presenta su monografía La anestesia general por vía intravascular (Figura 7) en la que expone su experiencia con el uso de varios anestésicos, al concurso convocado por el Instituto de España de la Real Academia Nacional de Medicina y que fue premiada con la "Medalla de Oro" por lo que recibió el título de académico corresponsal. Inicia una activa etapa investigadora, estudia, con una beca del Consejo Superior de Investigaciones Científicas, en la sección de fisiología del Instituto Ramón y Cajal y bajo la dirección del Dr. José María del Corral, la fisiopatología ureteral mediante cirugía experimental en animales, con cuyo resultado, Alteraciones dinámicas y morfológicas en el uréter consecutivo a la simpatectomía periureteral, demostró que la anastomosis término-terminal del uréter podía realizarse con éxito funcional, de tal modo que rehabilitó esta técnica quirúrgica y contribuyó al avance operatorio de las vías excretoras, por lo que fue premiado por la Real Academia Nacional de Medicina en 1941; también lo fue por la Beneficencia Provincial de Madrid, en 1943, por su trabajo sobre la exploración vésico-prostática por medio de la Cistografía lacunar, al inyectar aire y
RESUMEN PRACTICO

OE

DIAGNÓSTICO

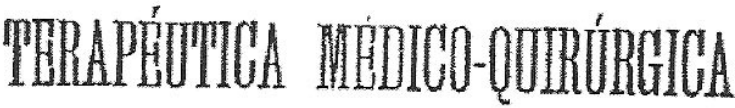

DE LA5

AFECGIONES DE LAS VIAS URINARIAS

Por

D. Rafael Mollá y Aodrigo

Doctor Isureado por la Universidad Central: Catedrutico nutherario, por oposiction, de Patologits y Clnie Quirúrgics en la Facultad de Afedicina de in Universidad de la Fabara

FIGURA 5. Resumen práctico de diagnóstico y técnica médico quirúrgica de la afecciones de las Vías urinarias, (Valencia, 1894), primer tratado de español de Urología escrito por el Dr. Mollá Rodrigo.

contraste en la vejiga, sistema que introdujo en España y que también publicó en el journal americano de Urología en 1950, y, en 1947, lo fue por La Uroquimografía, modalidad que permite, por medio de la superposición de placas radiológicas, apreciar el movimiento orgánico y que utilizó para visualizar el peristaltismo pielo-ureteral. Empleó por vez primera en nuestro país la vía tóraco-abdominal en una intervención de Nefrectomía transtorácica, en pacientes con cifoescoliosis, trabajo que también fue editado en revistas alemanas y efectuó la primera descripción en la literatura española de un tuberculoma renal. En el resto de sus trabajos, de sólida preparación, estudia y recoge datos de las principales patologías que atendía, se encuentran expuestos con claridad y limpieza en el lenguaje y acompañados por una extensa bibliografía, fruto de su meticulosidad, de su capacidad organizadora y de su gran laboriosidad. Tradujo del alemán la segunda edición del tratado del Dr. H. Boeminghaus, Urología. Terapéutica operatoria, clínica e indicaciones (Figura 9). 


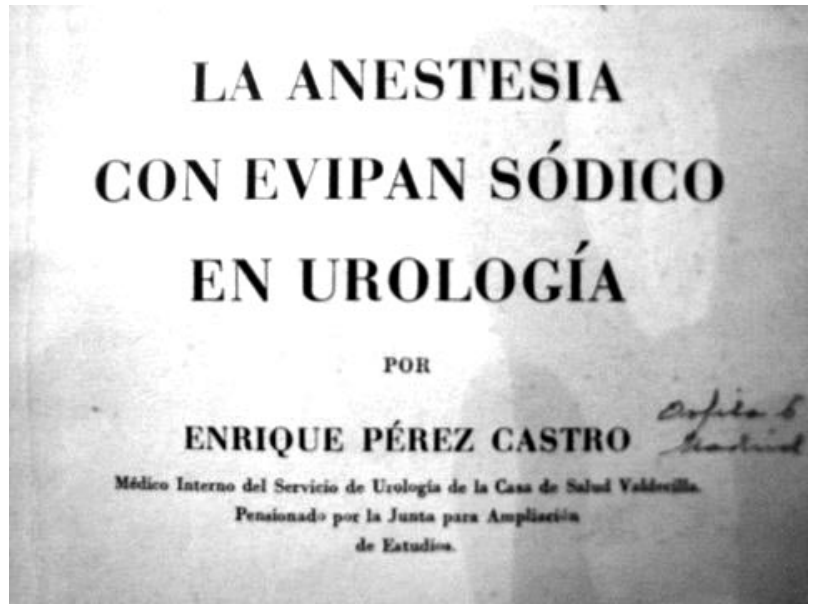

FIGURA 6. La anestesia con evipán sódico en Urología. Tesis Doctoral.

En 1944 accedió por concurso a la plaza de jefe clínico del Hospital Provincial y, a la jubilación del Dr. Pulido en 1949, obtuvo por oposición la jefatura del servicio al que se dedicó por entero (Figura 10) y logró levantarlo con la colaboración de un excelente equipo, entre ellos el Dr. Díez Yanguas, quien comenzó su labor en el centro en 1944 y en 1951 fue nombrado jefe clínico y le sucedió a su retiro al frente del servicio, $y$, desde 1953, con el Dr. Martínez Piñeiro y como ayudante el Dr. Amalio Pérez Coutiño. En 1968 se trasladó el Hospital del antiguo edificio de Atocha, que actualmente remodelado ocupa el museo Reina Sofía, a su actual ubicación, en el lugar donde estuvo situado el Hospital de San Juan de Dios, inaugurado con el nombre de Ciudad Sanitaria Provincial Francisco Franco (actual

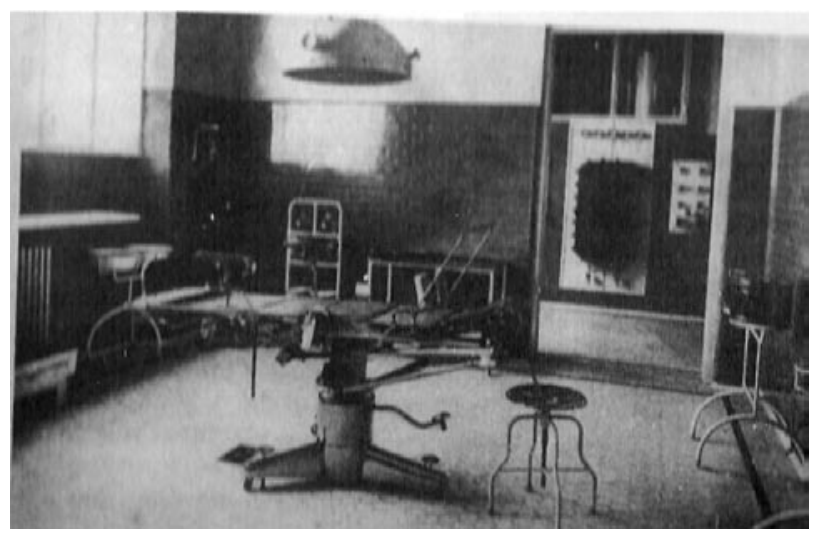

FIGURA 8.
TNATXTUTO DE TEFANA

necax A ond

LA ANESTESIA GENERAL POR VIA INTRAMUSCULAR TOR ET.

DR. D. ENRIQUE PEREZ CASTRO

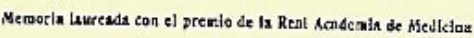
en el concirso de 1936. J7. - Tetra il.

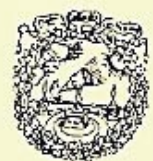

MADIRID

DIFRRTTA DR J. TRSANI

$F_{2}$ ) ras, 11.-Teld. $2005 \%$

19.1

FIGURA 7. La anestesia general por vía intravascular, trabajo premiado por el Instituto de España en 1940.

\section{U R O L O G I A}

TERAPEUTICA OPERATORIA, CLINICA E INDICACIONES

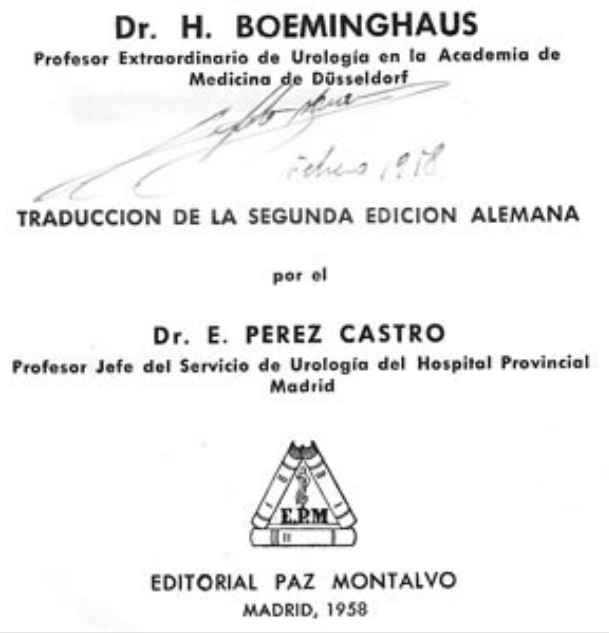

FIGURA 9. Tratado de Urología del Dr. Boeminghaus, traducción del alemán por el Dr. Pérez Castro, 1958. 


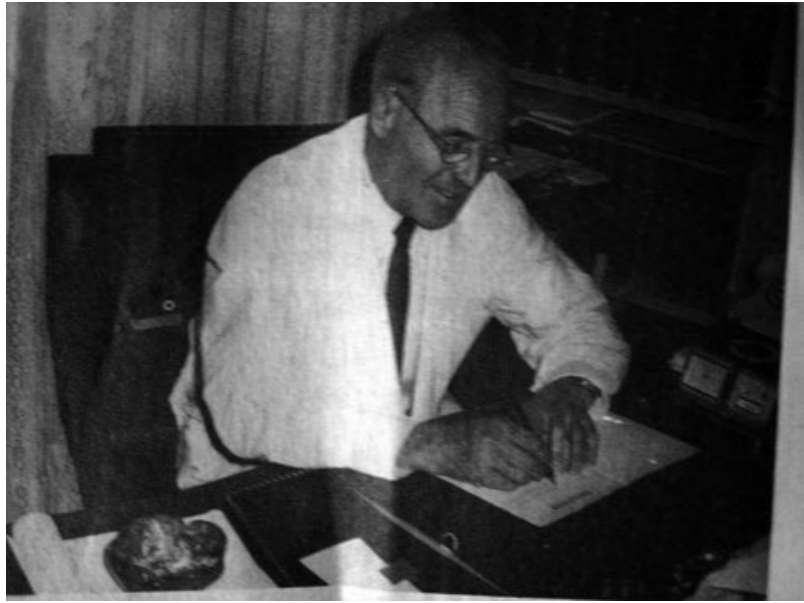

FIGURA 10. El Dr. Pérez Castro en su despacho del hospital.

Hospital General Universitario Gregorio Marañón); en él organizó cursos de postgrado, a los que invitaba a los profesores de mayor renombre, en 1969 al Dr. Yurii Yediny de Rubia, inventor del Urat 1, método de litotricia electrohidráulica para la destrucción de los cálculos renales; en 1970 celebró, junto con

Shade-1d, 4-III-69

ESTADTLWO NOUINAL DEG SERVICTO DB ORJTOGES

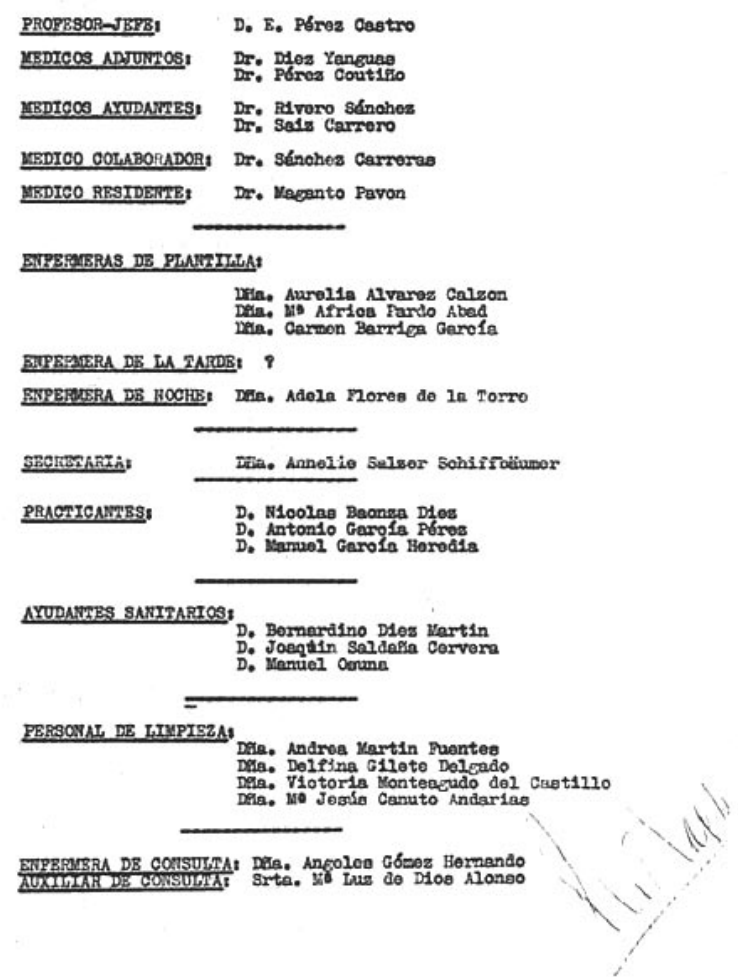

FIGURA 12. Miembros del Servicio de Urología en 1969.

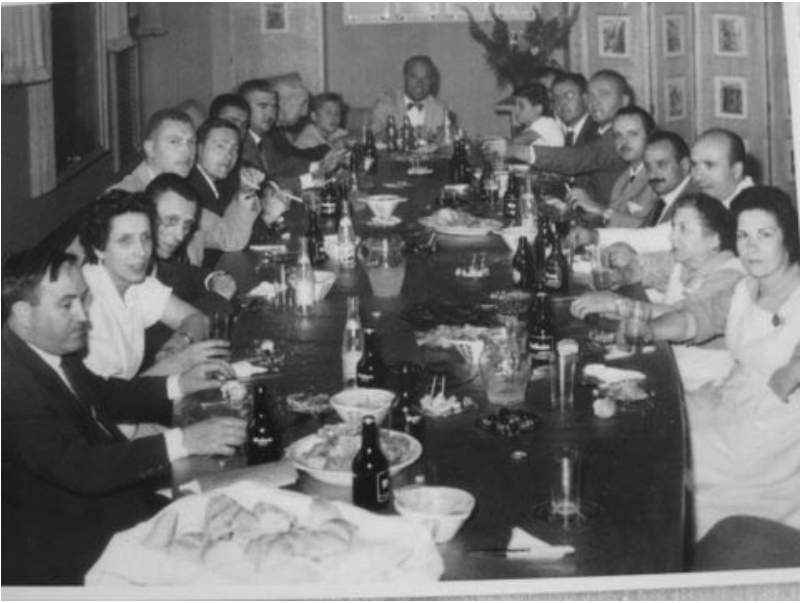

FIGURA 11. Grupo de miembros del servicio de Urología en una jornada de celebración de San Enrique.

el radioterapeuta Dr. Pérez Modrego, el primero de Actuación en Oncología Urológica. Acudieron a especializarse bajo su amparo a lo largo de los años los Drs. Antonio Valverde Mazuelas, Feliciano Román Ruíz, José Luis Sebastián Borruel, Eugenio Rivero Sánchez y Marcelino González Martín, en su primera etapa (Figura 11); posteriormente como médicos internos encontramos a José Rodríguez Cuadrado, Santiago Navío Niño, Ataúlfo Saiz Carrero y Emilio Maganto Pavón, entre otros, todos ellos aprendieron a sondar de la mano del practicante del servicio D. Bernardino Díaz Martín y conocieron su maestría en realizar dilataciones y sondajes casi imposibles (Figura 12). Perteneció a la Comisión Científica hospitalaria de la que fue secretario, su cometido consistía en recoger las comunicaciones presentadas por sus miembros en las sesiones clínicas para publicarlas en la revista editada por el centro, Hospital General. El Hospital Provincial se encontraba adscrito a la Facultad de Medicina de la Universidad Central en la que fue profesor ayudante de la cátedra de Terapéutica

\section{HOSPITAL GENERAL}

REVISTA DE MEDICINA Y CIRUGIA

BENEFICENCIA PROVINCIAL DE MADRID

Mayo-Junio 1977

$$
\begin{aligned}
& \text { Fundidot: C, GONZALEZ.BUENo } \\
& \text { Durecton: A. MUNoZ CALERo } \\
& \text { Rebactor JEFE: M. HIDALGo HUERT A } \\
& \text { SEchetanios of Redacción: J. M. PALACios mateos, } \\
& \text { E. PEREZ CASTRO Y E. FOLQUE GOMFZ }
\end{aligned}
$$

FIGURA 13. Hospital General, revista del Hospital Provincial de Madrid, donde figura como secretario de redacción. 
Clínica, a propuesta del Dr. Velázquez, y, en 1972, fue nombrado agregado de Urología (Figura 14). En 1978 se retiró de la vida docente y hospitalaria y falleció dos años más tarde.

Su principal obra la constituye la fundación, en 1944, de Archivos Españoles de Urología, (Figura 15) con la colaboración de Luis Cifuentes Delatte y Antonio Puigvert Gorro. Fue su promotor, editor y director durante 36 años, en los que se dedicó con entera pasión a la revista, su tesón fue el motor que hizo que la publicación se mantuviese con una activa pujanza y se alzara con el título de «principal órgano de difusión de la Urología española». Trabajador incansable, por las noches se recluía en su refugio-despacho, ubicado en una casita al fondo del jardín de su casa, para revisar los artículos, escribir los suyos, mantener correspondencia con los autores, solicitar originales, ordenar y disponer los trabajos, etc., además de poner al día las fichas de su magnífica biblioteca urológica, como refiere en el prólogo de la traducción del libro de Boemingaus, Los requerimientos acuciantes del ejercicio activo de nuestra especialidad llenan las horas de mi vida, he tenido,

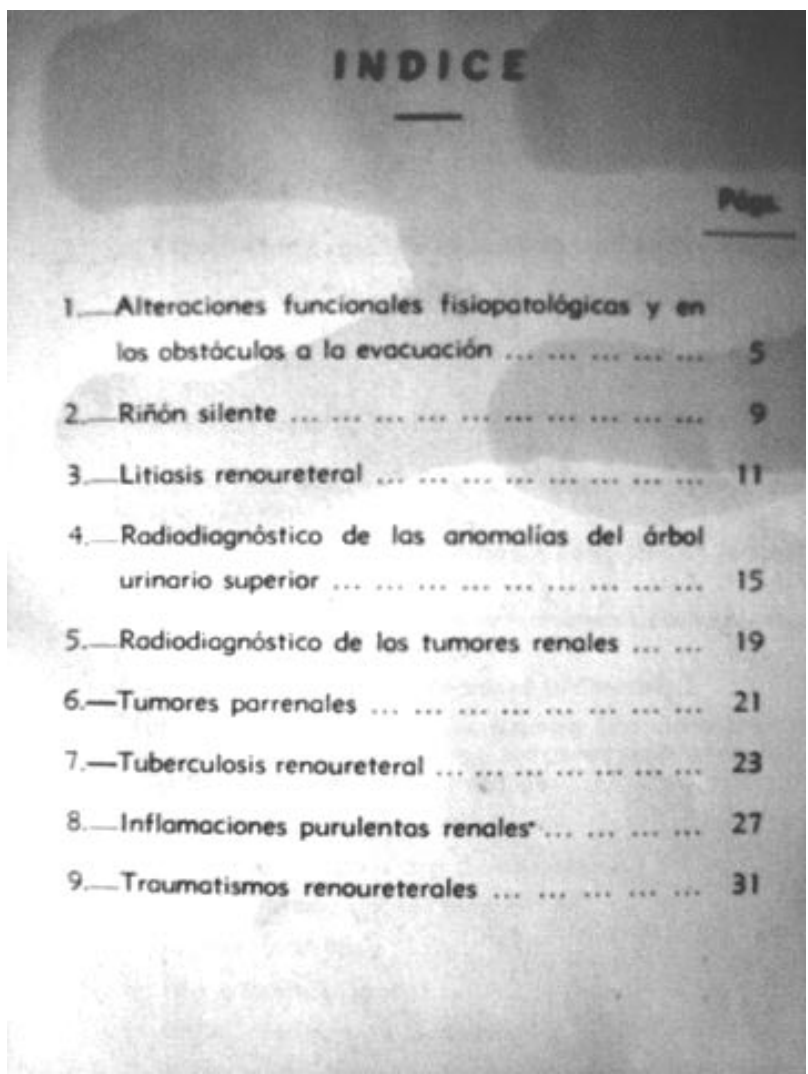

FIGURA 14. Programa para la docencia de los alumnos de la Facultad de Medicina. para dar cima a mi tarea, que robarle horas al bien ganado descanso.

Miembro de la Asociación Española de Urología, presentó un resumen de su tesis doctoral en la sesión celebrada en mayo de 1934, Resultados obtenidos en Urología con un nuevo anestésico general. Después de su reorganización tras la guerra y el restablecimiento de la actividad científica en 1945, fue secretario de actas y participó en la reunión del 16 de noviembre, con la que se inició el curso académico de 1945-1946, celebrada en el salón de Actos del Colegio de Médicos de Madrid bajo la presidencia de D. Pedro Cifuentes con la comunicación Duplicidad pieloureteral completa sin entrecruzamiento. lconografía en color. Desarrolló la ponencia del I Congreso lberoamericano y VII Hispano Portugués de Urología, que tuvo lugar entre Madrid y Barcelona, en junio de 1967, Litolisis en Urología (Figura 16). Perteneció a la Sociedad Internacional de Urología, nombrado en julio de 1947, de la que fue delegado

томо $\times X X I I I$

NUM. 6

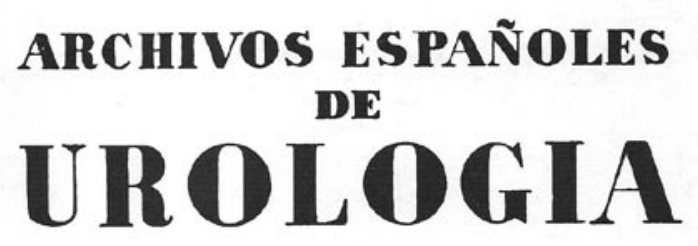

PUBLICADOS PO

E. PEREZ CASTRO, A. PUIGVERT GorRo y L. CIFUentes delatte

NEGROLOGICA

L. CIFUENTES DELATTE: Enrique Pérez Cantro (1908-1980).

ORIGINALES

E. PEREZ CASTRO: Antologia urológiea. Profeser Alexander von Lichtenberg (1830-1980) - B. LLOPIS, J. L. PERALES, M. GUILLEN y A. TRAMOYERES CA. SES: Carcinoma de urotelio superior. Aportación de 28 casos--L. IBARZ SERVIO, M. A. CALVO MATEO y J. I. IDOIPE TOMAS: Ectopias ureterales-A. LOPE2 PEREZ: Resultados lejanos de las ureteroileoplastias en cuanto a la función renalC. TORRES RANIREZ, J. AGUILAR RUIZ, A. ZULUAGA GOAEZ, R. ESPUELA ORGAZ, S. DEL RIO SAMPER y J. L. MARTINEZ TORRES: Cálculos renales de núcleo mixto--J. M. ${ }^{n}$ ARRUFAT, E. CERVELLo y F. ALBELL.A: Azoospermia excretora: Sindrome de Young.

Noticias

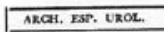

NOVIEMBRE-DICIEMBRE 1980

Revista regularmente ineluida en INDEX MEDICUS $y$ su MEDLINE DATA BASE

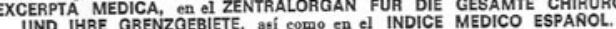

FIGURA 15. Portada de la revista Archivos Españoles de Urología, en el último número editado por él con la nota necrológica escrita por el Dr. Cifuentes. 
para España elegido por votación del capítulo español en 1965 y en la que efectuó una gran defensa de los intereses hispanos y de nuestro idioma con tenacidad y energía frente a la mayoría anglosajona. Fue socio de la belga, suiza e italiana y mantuvo siempre una excelente vinculación con la urología alemana por lo que fue condecorado por el Gobierno alemán con la Gran Cruz del Mérito Civil de primera clase. Invitado en numerosas ocasiones a Congresos como conferenciante, principalmente por compañeros de los países sudamericanos con quienes le unía una excelente relación (Figura 17), en 1963 estuvo como representante del Instituto de Cultura Hispánica, en el IX Congreso Panamericano, en Bogotá; fueron numerosos los urólogos hispanoamericanos que asistían, para ampliar conocimientos, a su servicio.

Amante de los libros, se deleitaba con una buena lectura, amigo fiel y conversador amable, su tiempo libre lo repartía con su familia y con sus aficiones, especialmente la caza y la observación de la na-

III. NUMERO EXTRAORDINARIO

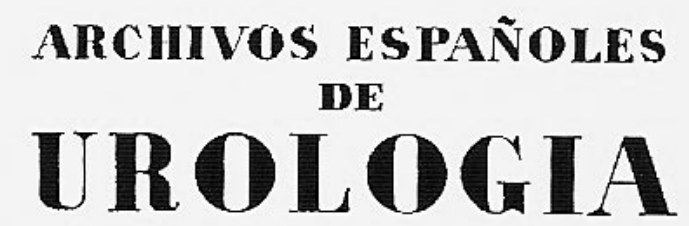

URGANO DE LA ASOCLACION ESPAÑOLA DE UROLOGIA rtiLtCADO HOR.

E. PFIRF: CASH'RO, A. PUIGVERE GORRO Y T. CIEUENTES DFT.ATTV

LITOLISIS FN UROLOGIA

De. Enrique Pẹ́res Ciastro

Ponencia del I Congreso Iberoamericano y VII Hispanu-Portugués de Urología, Madrld-Barcelona, 27-VI-fiz y 5-VII-67

FIGURA 16. Litolisis en Urología, ponencia del I Congreso Ibero Americano de Urología, 1967.

turaleza y se extasiaba ante un espléndido paisaje. Falleció en Madrid, el 16 de noviembre de 1980.

Entresacamos, de su extensa bibliografía, las publicaciones que estimamos de mayor interés:

- Consideraciones sobre un caso de pionefrosis, Rev. Esp. Uro., I: 66-73, Madrid, 1933.

- Die klinischen chemischen und morphologisches Folgen des Kochsalzmangels in Blut, Deutsche Medizinische Wochenschrift, 19, 743, 1937.

- La anestesia general por vía intravascular, tesis doctoral, 82 págs. Impr. J. Cosano, Madrid, 1941.

- Trastornos de la micción en la hipertrofia prostática, Clínica y Laboratorio, febrero, Madrid, 1942.

- Cistografía lacunar, premiado por la Beneficencia Provincial, Rev. Clín. Esp., XI: 175, 1943.

- Empleo de los rayos X en la patología próstata vesical, Rev. Clín. Esp. IV (10): 3, 1943.

- La cistografía lacunar y los tumores papilares de vejiga, Gaceta Médica Española, mayo: 144, 1944.

- Alteraciones dinámicas y morfológicas en el uréter consecutivo a la simpatectomía periureteral, Arch. Esp. Urol., I (1): 36-68, 1944.

- Nuevo estudio del adenoma periuretral y de la llamada celda prostática, Arch. Esp. Urol., l (2): 123139, 1944.

- Agenesia total sacrocoxígea y sus repercusiones urológicas, Cirugía del aparato locomotor, I: 104, 1944.

- Tuberculosis renal productiva de forma pseudo tumoral. Rev. Esp. Tuberculosis, XIII (112): 106, julio 1944.

- Tuberculoma renal, Arch. Esp. Urol., II, (1): 59-76, 1945.

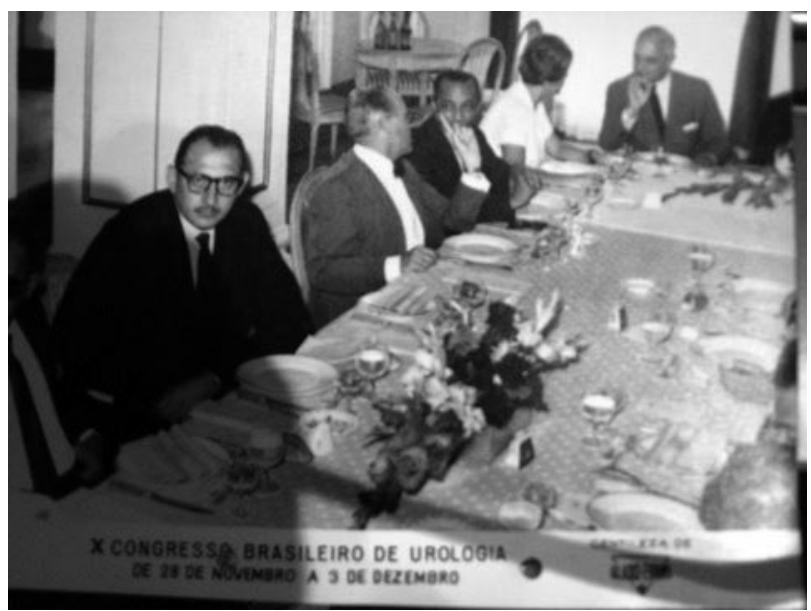

FIGURA 17. En el X Congreso Brasileño de Urología. 
- Epitelioma de uretra (fosa navicular), Arch. Esp. Urol., II, (6): 140-153, 1945.

- Duplicidad pieloureteral completa sin entrecruzamiento, Rev. Esp. Cirug. II (9): 190-193 sept. 1945.

- Ureteritis quística, Arch. Esp. Urol., III, (1): 43-62, 1946.

- Quiste seroso de riñón Rev. Esp. Cirug. III (16): 301, 1946.

- Concepto actual del reflujo vésico ureteral, Arch. Esp. Urol., III (2): 122-129, 1946.

- Uroquimografía, premiado por la Beneficencia Provincial de Madrid, Arch. Esp. Urol., IV, (4): 275-317, 1947.

- Perforación calculosa de las vías urinarias superiores, Rev. Esp. Cirug., V (26): 123-128, febrero de 1948.

- Sutura total de vejiga sin sonda permanente en la talla por cálculos, Rev. Clín. Esp. XXIX (6): 381-387, 1948.

- Sutura totale della vescica senza catetere a permanenza nella cistostomia per calcolo, Urología, XV (I), junio de 1948.

- In memoriam: Prof.Alexander von Lictenberg, Arch. Esp. Urol., VI (2): 91, 1950.

- Perforacione da calcolo delle vie urinarie superiore, Urologia, XVII (III), junio 1950.

- Diagnostic value of lacunar cystography, Journal of Urology, 64 (3), sep. 1950.

- Formas singulares de la tuberculosis urinaria, Urología, XVIII (II), abril 1951.

- Estrangulación del pene, Arch. Esp. Urol., VIII (1): $1-9,1952$.

- Nefrectomía transtorácica, Arch. Esp. Urol., VIII (2): 97-121, 1952.

- Uréter retrocava, Arch. Esp. Urol., VIII (3): 183199, 1952.

- Tumor dermoide vesical, Arch. Esp. Urol., VIII (4): 291-314, 1952.

- Transthorakale nephektomie, Zeitschift für Urologie, 470: 51 -54, 1957.

- Valor diagnóstico del Papanicolau en los tumores mínimos del aparato urinario, Arch. Esp. Urol., XVI (2): 109-1 18, 1963.

- Cirugía en las fusiones renales, Arch. Esp. Urol., XVI (4): 313-332, 1963.

- Perforación del aparato urinario de origen esquelético, Arch. Esp. Urol., XVII (4): 31 1-320, 1964

- Influjos endocrinos en la vía excretora urinaria, Arch. Esp. Urol., XVIII (3): 169-188, 1965.

- Bazo heterotópico paraepididimario, Arch. Esp. Urol., XIX (2): 140, 1966.

- Disfunciones vesicales neurógenas en la agenesia sacrocoxígea, Arch. Esp. Urol., XIX (3): 267-270, 1966.

- Litolisis en Urología, 150 págs., ponencia I Congreso lbero Americano de Urología, Arch. Esp. Urol., XX (2): 109, III, núm. extra, 1967.
- La vejiga diabética, Hospital General, VII (5), 1967.

- In memoriam: profesor Dr. D. Ángel Pulido Martín, Arch. Esp. Urol., XXIII (4): 319, 1970.

- Destrucción de los cálculos vesicales mediante impulsos eléctricos, homenaje al profesor B. Lorenzo Velázquez, edit. Oteo, Madrid, 1971.

- Hemangiomas vesicales juveniles, Arch. Esp. Urol., XXVII (5): 575-588, 1974.

- Uréter ciego craneal, Arch. Esp. Urol., XXVIII (1): 1-32, 1975.

- Quistografía renal. Comentarios sobre su reactualización, Arch. Esp. Urol., XXIX (1): 1-36, 1976.

\section{AGRADECIMIENTOS}

Al Dr. Pérez-Castro Ellendt por revisar el original y facilitarme datos y fotografías.

Al Dr. Saiz Carrero por su aportación de la relación de los componentes del servicio.

\section{BIBLIOGRAFÍA y LECTURAS RECOMENDADAS (*lectura de interés $y^{* *}$ lectura fundamental)}

1. CIFUENTES DELATTE, L.: "Enrique Pérez Castro". Arch. Esp. Urol., 23: 523, 1980.

*2. IBID.: "La clínica urológica de Von Lichtenberg en Berlín (1933)”. Arch. Esp. Urol., 48: 105, 1995.

3. INSAUSTI CORDÓN, J.L.: "Enrique Pérez Castro". Actas Uro. Esp., 5, 1981.

4. IBID.: "Enrique Pérez Castro", Compendio histórico de la Urología Española y de su Asociación, ponencia IV Congreso Hispano Americano de Urología, pág. 97, Acapulco, 1982.

*5. MAGANTO PAVÓN, E.: "La Urología en Madrid", Historia Biográfica y Bibliográfica de la Urología Española en el siglo XX, pág. 221, Edicomplet, Madrid, 2002.

6. MARTÍNEZ PIÑERO, J.A.: "In memorian". Arch. Esp. Urol., 34: 1, 1981.

*7. PÉREZ ALBACETE, M.: "Cien figuras de la Urología Española". Tip. San Francisco, Murcia, 2005.

8. PUIGVERT GORRO, A.: "Dr. D. Enrique Pérez Castro". An. Fund. Puigvert, 11: 42, 1981.

9. IBID.: "E. Pérez Castro". Arch. Esp. Urol., 34: 3, 1981.

**10. SAIZ CARRERO, A.: "La Urología en Madrid". Historia Biográfica y Bibliográfica de la Urología Española en el siglo XX, pág. 265, Edicomplet, Madrid, 2002. 